\title{
Coupled dynamics of interacting spin-1 bosons in a double-well potential
}

\author{
D. W. S. Carvalho, A. Foerster, and M. A. Gusmão \\ Instituto de Fisica, Universidade Federal do Rio Grande do Sul, Caixa Postal 15051, 91501-970 Porto Alegre, Brazil
}

(Received 3 January 2018; published 21 March 2018)

\begin{abstract}
We present a detailed analysis of dynamical processes involving two or three particles in a double-well potential. Motivated by experimental realizations of such a system with optically trapped cold atoms, we focus on spin-1 bosons with special attention on the effects of a spin-dependent interaction in addition to the usual Hubbard-like repulsive one. For a sufficiently weak tunneling amplitude in comparison to the dominant Hubbard coupling, particle motion is strongly correlated, occurring only under fine-tuned relationships between well-depth asymmetry and interactions. We highlight processes involving tunneling of coupled particle pairs and triads, emphasizing the role of the spin-dependent interaction in resonance conditions.
\end{abstract}

DOI: 10.1103/PhysRevA.97.033615

\section{INTRODUCTION}

In the past decades, fast development of techniques for trapping and cooling atoms to very low temperatures in optical lattices allowed for experimental realization of a number of lattice models of fermionic or bosonic interacting particles [1-4]. Nowadays, it is possible to build from many-body systems in large lattices to few particles in few wells with a high degree of control over well depths, interwell tunneling amplitude, and strength of on-site interactions. Furthermore, an important research avenue was opened by the possibility of having Bose-Einstein condensates with nonfrozen spin degrees of freedom [4-8]. Both spin [9] and particle [10] dynamics for large spinor condensates in a double well have been discussed.

The experimental ability to isolate a limited number of atoms in few wells [11-19] attracted attention to few-particle systems $[20,21]$. In this context, theoretical descriptions of ground-state properties of few spin-1 bosons in double wells were recently reported $[22,23]$. The study of dynamics in such systems is also of great interest since it involves the controlled evolution of strongly entangled states, which is relevant for possible applications in atomtronics [24-26] and quantum computing. Experiments on this line have been performed with both fermionic [27,28] and bosonic atoms [29-32], revealing effects such as interaction blockade and pair tunneling.

In this paper we present a theoretical investigation on the dynamical evolution of two and three spin-1 bosons in a double-well potential, described by a variant of the twosite Bose-Hubbard Hamiltonian [33] that includes a spindependent interaction [34] in addition to the usual repulsive Hubbard- $U$ term. We pay special attention to fine-tuning of different regimes, like conditional single-particle tunneling, and the motion of bound pairs and triads. Such processes have been experimentally observed for spinless bosons [29-32], and our main goal is to describe effects of spin-dependent interaction that could also be detectable in experiments.

The paper is organized as follows. In Sec. II we present the Hamiltonian and discuss an appropriate basis to represent the relevant quantum states. We revisit the spinless case for the two-particle system in Sec. III, exploring some features that are important to the subsequent development. Sections IV A and IV B, respectively, are devoted to discussions of two- and three-particle dynamics taking spin into account. There we analyze the interplay between double-well asymmetry (bias) and interactions. Final remarks are presented in Sec. V.

\section{MODEL}

Bosonic atoms with nonzero spin trapped in a doublewell optical potential may be described by a two-site BoseHubbard model extended to include a spin-dependent interaction $[22,23]$. The Hamiltonian is written as

$$
\begin{aligned}
H= & \sum_{i=L, R} \epsilon_{i} n_{i}-J \sum_{\sigma}\left(a_{L \sigma}^{\dagger} a_{R \sigma}+a_{R \sigma}^{\dagger} a_{L \sigma}\right) \\
& +\frac{1}{2} U \sum_{i=L, R} n_{i}\left(n_{i}-1\right)+\frac{1}{2} U^{\prime} \sum_{i=L, R}\left(\mathbf{S}_{i}^{2}-2 n_{i}\right),
\end{aligned}
$$

where $a_{i \sigma}^{\dagger}$ and $a_{i \sigma}$ are creation and annihilation operators of a boson in the single bound state of a given well, labeled by $L$ (left) and $R$ (right) in a spin state $\sigma$; the operators associated with the number of bosons and total spin at each well are denoted by $n_{i}$ and $\mathbf{S}_{i} ; J$ is the tunneling parameter (or hopping constant); $U$ is the usual (repulsive) Hubbard interaction, and $U^{\prime}$ measures a local spin-dependent interaction. We will restrict our analysis to spin- 1 bosons so that $\sigma$, the quantum number associated with a single-particle spin component $S^{z}$, can assume the values of $\{1,0,-1\}$. Essentially all the Hamiltonian parameters can be tuned in optical-lattice realizations of this model $[29,30,35]$. We consider a site-dependent local energy, allowing for asymmetrical wells, which could be viewed as the effect of a bias potential. The spin coupling constant $U^{\prime}$ can be positive or negative, favoring low- and high-spin states, respectively. Experimentally, the $U^{\prime} / U$ ratio is uniquely determined from the difference in scattering length between spin channels of two trapped atoms. For example, $U^{\prime} / U \simeq$ 0.04 for ${ }^{23} \mathrm{Na}$ and -0.01 for ${ }^{87} \mathrm{Rb}[6]$.

The total number of particles $\left(N_{T}=N_{L}+N_{R}\right)$, the total $\operatorname{spin}\left(\mathbf{S}_{T}=\mathbf{S}_{L}+\mathbf{S}_{R}\right)$, as well as any component of the latter are conserved quantities. This can be used to restrict the analysis to subspaces with definite eigenvalues of $N_{T}, \mathbf{S}_{T}^{2}$, and $S_{T}^{z}$. Our 
choice of basis states for matrix representations will be a set of vectors of the form $\left|\left\{N_{L}, N_{R}\right\},\left\{S_{L}, S_{R}\right\}, S_{T}\right\rangle$, which have welldefined occupation numbers and total spins in each well $\left(S_{i}\right.$ is the quantum number associated with the eigenvalues of $\mathbf{S}_{i}^{2}$ ). It should be noted that state-vector symmetry with respect to particle exchange implies that the sum $S_{i}+N_{i}$ is always equal to an even integer [22]. We have omitted the eigenvalue of $S_{T}^{z}$ in our basis because the Hamiltonian is rotationally invariant in spin space. In practice, we always choose the zero eigenvalue, which is present in all subspaces of definite $S_{T}$.

In the absence of interwell tunneling the Hamiltonian is diagonal in the basis just defined. Thus, the hopping constant $J$ enters only in off-diagonal elements of the $H$ matrix. In order to determine the correct coefficients of $J$ in such matrix elements, one needs to write the basis states in terms of vectors that specify the occupation number $N_{i}^{\sigma}$ of each spin state in each site. Due to our restriction to states with zero $S_{T}^{z}$, we only have to choose sets of occupation numbers for which the sum of all $\sigma$ 's is equal to zero.

\section{TWO-PARTICLE DYNAMICS FOR $U^{\prime}=0$}

Our analysis focuses on the dynamics of few bosons in a double well. In the spinless case, this has been the subject of previous investigations [29-31,36,37]. It is then convenient to begin with $U^{\prime}=0$, the usual Bose-Hubbard model, for which analytical solutions are easily obtained, at least for one and two particles. In this limit the spin is irrelevant, and the basis vectors only need to specify the number of particles in each well, i.e., $\left|N_{L}, N_{R}\right\rangle$.

For a single particle in the system, there are two possible states $|1,0\rangle$ and $|0,1\rangle$, which are connected by the off-diagonal matrix elements $-J$. The transition between these states is maximized in the unbiased case $\left(\epsilon_{L}=\epsilon_{R}\right)$ due to energy conservation. In this regime, if we begin with the particle on the left (initial-state $|1,0\rangle$ ), the probability of finding it on the right at any time is given by

$$
|\langle 0,1|\mathcal{T}(t)| 1,0\rangle|^{2}=\frac{1}{2}[1-\cos (2 J t)]
$$

where $\mathcal{T}(t)=\exp (i H t)$ is the time-evolution operator (with the choice of $\hbar=1$ ). We thus see that the particle goes back and forth between the wells with an angular frequency $2 J$ (frequency $J / \pi$ ).

With two particles, there are three basis states: $|2,0\rangle,|0,2\rangle$, and $|1,1\rangle$. The states with a doubly occupied well are connected to the one with separate particles by a matrix element $-\sqrt{2} J$. At zero bias, starting with both particles on the left, the probability $P_{1,1}$ of finding them in separate wells is

$$
P_{1,1}(t)=|\langle 1,1|\mathcal{T}(t)| 2,0\rangle|^{2}=\frac{4 J^{2}}{\alpha^{2}}[1-\cos (\alpha t)]
$$

where $\alpha=\sqrt{U^{2}+16 J^{2}}$. Note that the amplitude depends on $J$ and $U$, in contrast with Eq. (2).
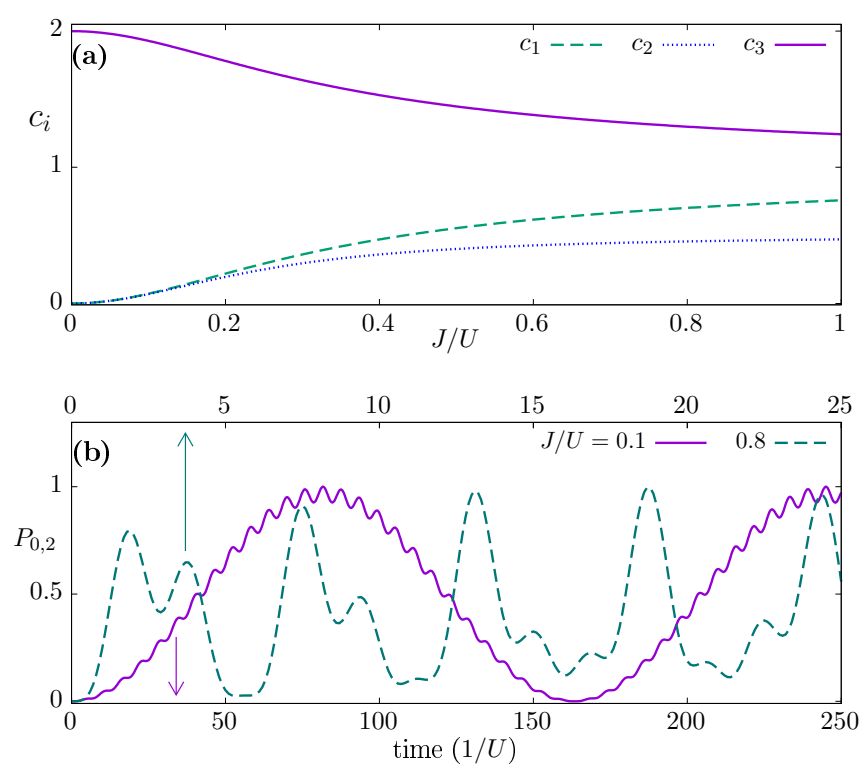

FIG. 1. (a) Amplitude $c_{i}$ of each oscillation component for pair transition, defined in Eq. (4), as a function of tunneling strength. (b) Time evolution of the probability of finding both particles on the right-hand side for two different tunneling regimes. Note that the time scales of the two curves differ by a factor of 10 .

For the same initial condition, the probability $P_{0,2}$ of finding both particles on the right-hand side is given by

$$
\begin{aligned}
P_{0,2}(t)= & |\langle 0,2|\mathcal{T}(t)| 2,0\rangle|^{2} \\
= & \frac{1}{4}\left\{1+\frac{U^{2}+8 J^{2}}{\alpha^{2}}-c_{1} \cos \left[\frac{1}{2}(\alpha+U) t\right]\right. \\
& \left.+c_{2} \cos (\alpha t)-c_{3} \cos \left[\frac{1}{2}(\alpha-U) t\right]\right\},
\end{aligned}
$$

with the coefficients of the oscillating terms defined as $c_{1}=$ $1-U / \alpha, c_{2}=8 J^{2} / \alpha^{2}, c_{3}=1+U / \alpha$. Figure 1(a) shows how these coefficients vary with the ratio $J / U$. Although we do not have a unique frequency for any values of $J$ and $U$, we see that essentially only the $c_{3}$ term survives in the stronginteraction regime $(J / U \ll 1)$. The dominant frequency is then

$$
\omega=\frac{1}{2}(\alpha-U) \simeq 4 J^{2} / U
$$

This is shown as the slowly varying curve of $P_{0,2}$ for $J / U=$ 0.1 in Fig. 1(b). Superimposed we observe a high-frequency contribution with very low amplitude, corresponding to the $c_{1}$ and $c_{2}$ terms in Eq. (4), whose frequencies are both nearly equal to $U$. For comparison, Fig. 1(b) also shows the behavior of $P_{0,2}$ when $J / U=0.8$ in which case the presence of three competing frequencies yields a substantially different time dependence.

It is interesting to remark that the probability of finding one particle in each well, Eq. (3), is strongly suppressed in the limit $U \gg J$ due to the large energy difference between states $|2,0\rangle$ and $|1,1\rangle$. Furthermore, in this limit the dominant terms in Eq. (4) reproduce the single-particle form of Eq. (2) but with the frequency $2 J$ replaced by $\omega$ as given by Eq. (5). Therefore, we have both particles tunneling as a bound pair with an effective 
hopping constant $\tilde{J}=2 J^{2} / U$. Such pair hopping has been observed experimentally, both in double wells [30] and in larger lattices [29].

An interesting effect arises from the introduction of a bias potential through a nonzero value of the reference energy of the right-hand side, $\epsilon_{R}$. It contributes to the diagonal matrix elements of the Hamiltonian, affecting frequencies and amplitudes in Eqs. (3) and (4). Its most remarkable effect is that single-particle tunneling, suppressed for two strongly interacting particles at zero bias (interaction blockade), is fully recovered for $\epsilon_{R}=U$. Conversely, a single particle cannot tunnel between wells of unequal depths unless there is another particle in the deeper well and their interaction exactly balances the energy mismatch. This is the conditional tunneling discussed in Ref. [30].

\section{DYNAMICS FOR $U^{\prime} \neq 0$}

We now turn to a more general analysis of dynamic processes, taking into account the spin-dependent interaction. We first discuss a two-particle system, which can be straightforwardly related to the spinless case of the last section.

\section{A. Two-particle system}

Conservation of total spin allows us to utilize the results obtained for $U^{\prime}=0$. This is possible due to a one-to-one correspondence between the basis vectors for a given $S_{T}$ and those of the spinless case,

$$
\begin{aligned}
& |0,2\rangle \longleftrightarrow\left|\{2,0\},\{2,0\}, S_{T}\right\rangle, \\
& |1,1\rangle \longleftrightarrow\left|\{1,1\},\{1,1\}, S_{T}\right\rangle, \\
& |0,2\rangle \longleftrightarrow\left|\{0,2\},\{0,2\}, S_{T}\right\rangle,
\end{aligned}
$$

which is valid for $S_{T}=0$ or 2 . These are the only $S_{T}$ values that yield any dynamics due to the condition that the sum of total spin and occupation number in each well must be even. The state correspondences in Eq. (6) are accompanied by the replacements $U \rightarrow U-2 U^{\prime}$ for $S_{T}=0$ and $U \rightarrow U+U^{\prime}$ for $S_{T}=2$ in the energy eigenvalues, likewise affecting Eqs. (3) and (4).

The strong-coupling regime is still characterized by $J / U \ll$ 1 , provided that $\left|U^{\prime}\right| \ll|U|$, which is true for the most commonly used atoms, such as ${ }^{23} \mathrm{Na}$ and ${ }^{87} \mathrm{Rb}$. In this regime, the frequencies of bound-pair oscillation for $S_{T}=0$ and 2 are

$$
\begin{gathered}
\omega_{S 0}=\frac{1}{2}\left[\sqrt{\left(U-2 U^{\prime}\right)^{2}+16 J^{2}}-\left(U-2 U^{\prime}\right)\right], \\
\omega_{S 2}=\frac{1}{2}\left[\sqrt{\left(U+U^{\prime}\right)^{2}+16 J^{2}}-\left(U+U^{\prime}\right)\right] .
\end{gathered}
$$

Figure 2(a) shows how these two frequencies vary in a narrow region of $U^{\prime} / U$ containing the values corresponding to ${ }^{23} \mathrm{Na}$ and ${ }^{87} \mathrm{Rb}$. The other two panels of Fig. 2 present the time evolution of population imbalance $\langle N I\rangle$, defined as $\left(\left\langle N_{R}\right\rangle-\right.$ $\left.\left\langle N_{L}\right\rangle\right) / N_{T}$. For realistic ratios $U^{\prime} / U$ the frequency change with respect to the spinless case is more pronounced for ${ }^{23} \mathrm{Na}$ in the $S_{T}=0$ channel as seen in Fig. 2(c).

We now study the combined effects of spin-dependent interaction and interwell bias in a broader range of values, regardless of experimental restrictions on them. We do this by
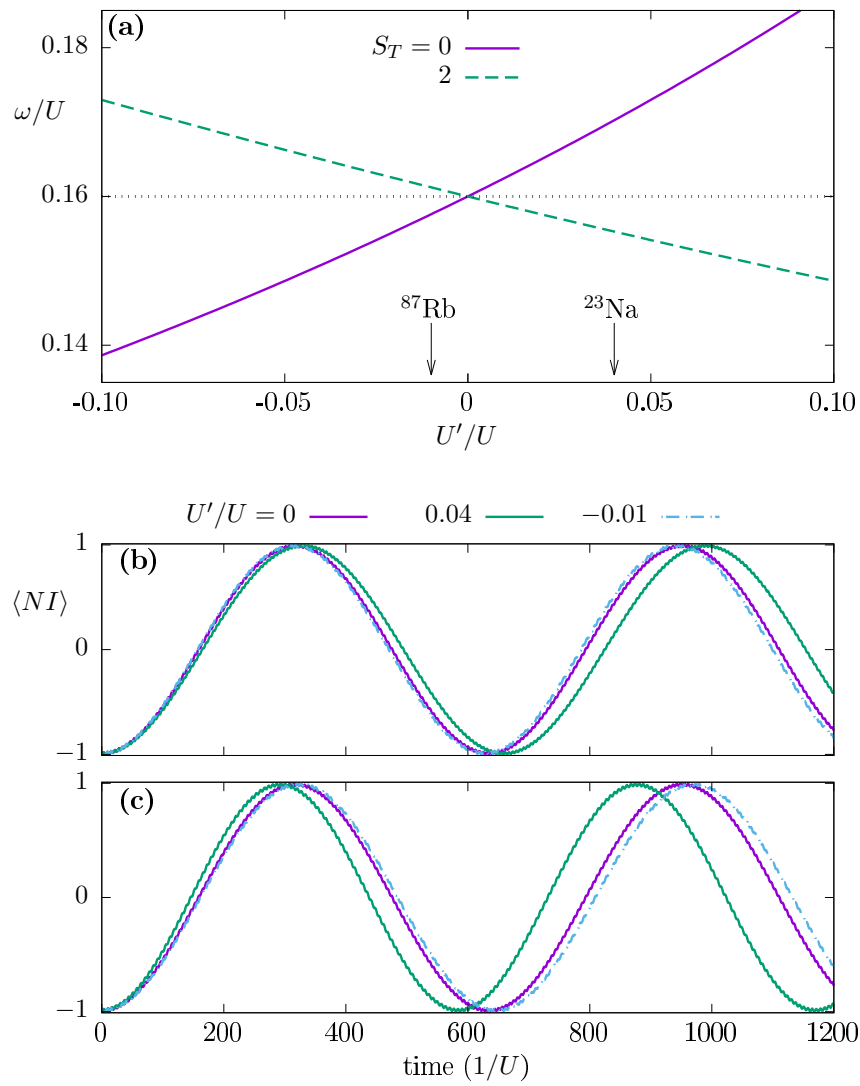

FIG. 2. (a) Variation of the pair-tunneling frequency of two spin-1 bosons in a symmetric double well for small values of $U^{\prime} / U$ in both $S_{T}$ channels. This frequency change is observed in the time evolution of population imbalance as shown in (b) and (c), respectively, for $S_{T}=2$ and 0 with the choice of $J / U=0.05$ and the indicated values of $U^{\prime} / U$ (corresponding to ${ }^{23} \mathrm{Na}$ and ${ }^{87} \mathrm{Rb}$ ).

evaluating the average number of particles $\left\langle N_{R}\right\rangle$ in the initially empty well as a function of time. It oscillates between the limits zero and two, involving both probabilities of having a nonzero occupation of the right-hand-side well, Eqs. (3) and (4). Fixing $U$ as the energy scale and choosing a small tunneling amplitude, we use the maximum value $\left(M_{R}\right)$ attained by $\left\langle N_{R}\right\rangle$ to construct the $U^{\prime}$ vs $\epsilon_{R}$ diagrams shown in Fig. 3 . For each set of parameters, we let the system evolve during a time sufficiently long to guarantee that at least one period was elapsed. Note that for both $S_{T}=0$ and 2 there is a line at zero bias for which $M_{R} \simeq 2$, corresponding to bound-pair tunneling. The other two lines, whose positions and angles change with the $S_{T}$ value, correspond to $M_{R} \simeq 1$, reflecting the single-particle conditional tunneling. These lines are determined by the relationship between $U^{\prime} / U$ and $\epsilon_{R} / U$ that ensures bias compensation of the interaction blockade in each case. All processes become resonant at the crossing points. Note that conditional-tunneling lines (for both $S_{T}$ values) contain the point $\left(U^{\prime}=0, \epsilon_{R}=U\right)$, in agreement with our comment at the end of Sec. III. Note that the line on which $M_{R} \simeq 1$ is wider. This can be viewed as off-resonance trips of one atom to the other well (equivalent to barrier penetration by the wave function), which is more noticeable for first-order tunneling. 
(a)

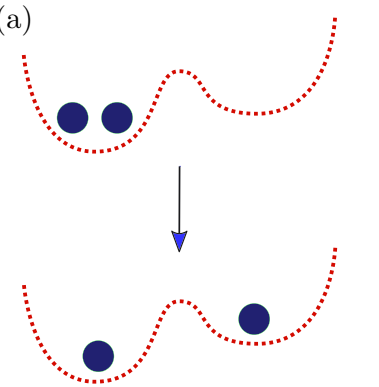
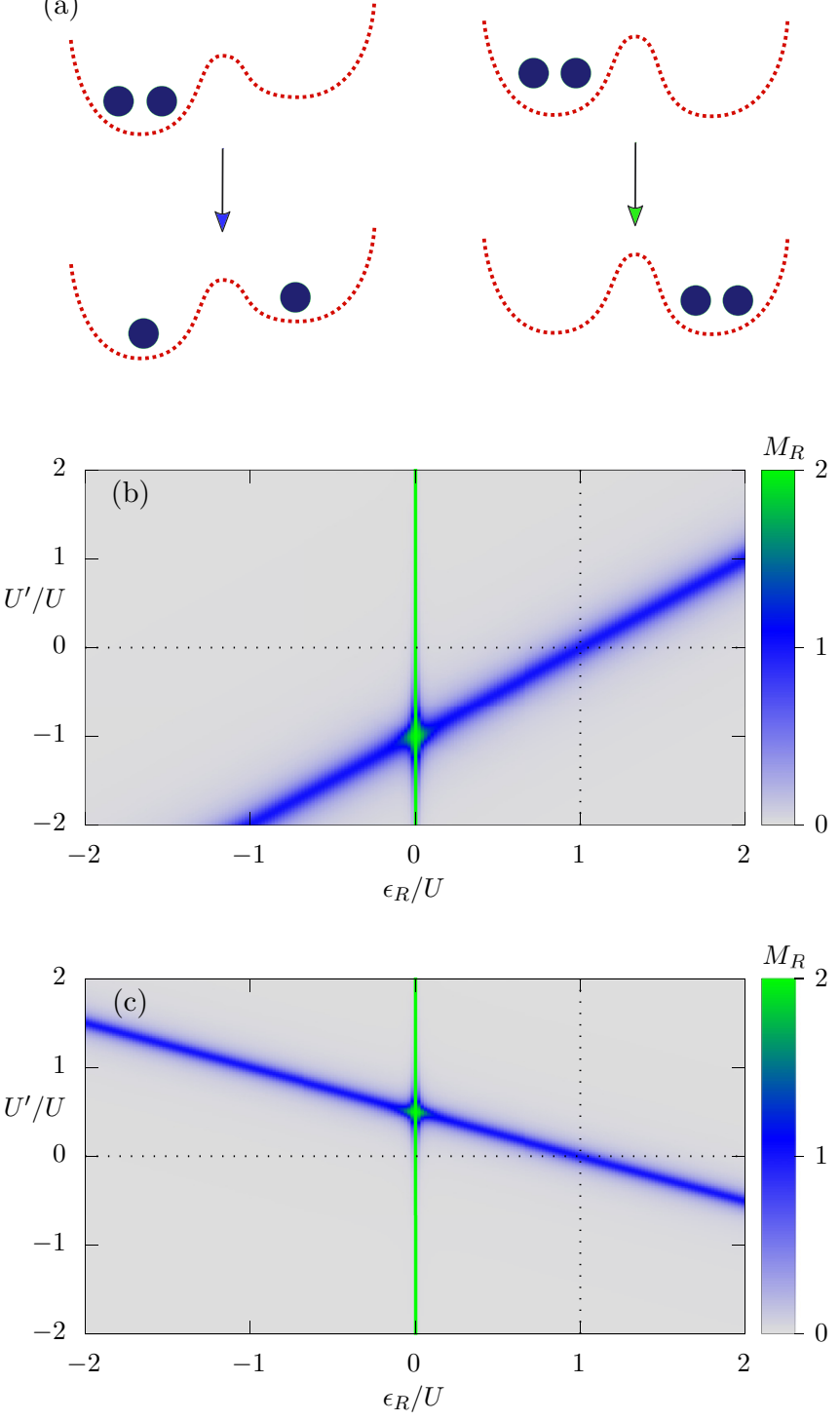

FIG. 3. Dynamics of two spin-1 bosons in a double well starting with both on the left as schematically represented in (a). The diagrams in (b) and (c), respectively, for $S_{T}=2$ and $S_{T}=0$ are intensity maps of the maximum average occupation $M_{R}$ of the right-hand side after a long evolution time $J / U=0.05$. The narrow colored regions in the $\left(\epsilon_{R}, U^{\prime}\right)$ parameter space reflect resonance conditions for singleparticle (blue) and pair (green) tunneling processes.

\section{B. Three-particle system}

Adding a third particle, we may have $S_{T}=3$ or 1 when all of them are in the same well. Consequently, two initial states are possible with all particles on the left-hand side. Here we use a fully numerical solution, diagonalizing the Hamiltonian matrix to obtain energy eigenvalues and eigenvectors, with which any time-dependent average can be evaluated. Nevertheless, the observed dynamics may be qualitatively interpreted in the weak-tunneling regime with an analysis of the kind employed in the previous subsection.

We first discuss the high-spin case. This subspace has four basis states $|\{3,0\},\{3,0\}, 3\rangle,|\{2,1\},\{2,1\}, 3\rangle,|\{1,2\},\{1,2\}, 3\rangle$, and $|\{0,3\},\{0,3\}, 3\rangle$ in the notation $\left|\left\{N_{L}, N_{R}\right\},\left\{S_{L}, S_{R}\right\}, S_{T}\right\rangle$. At
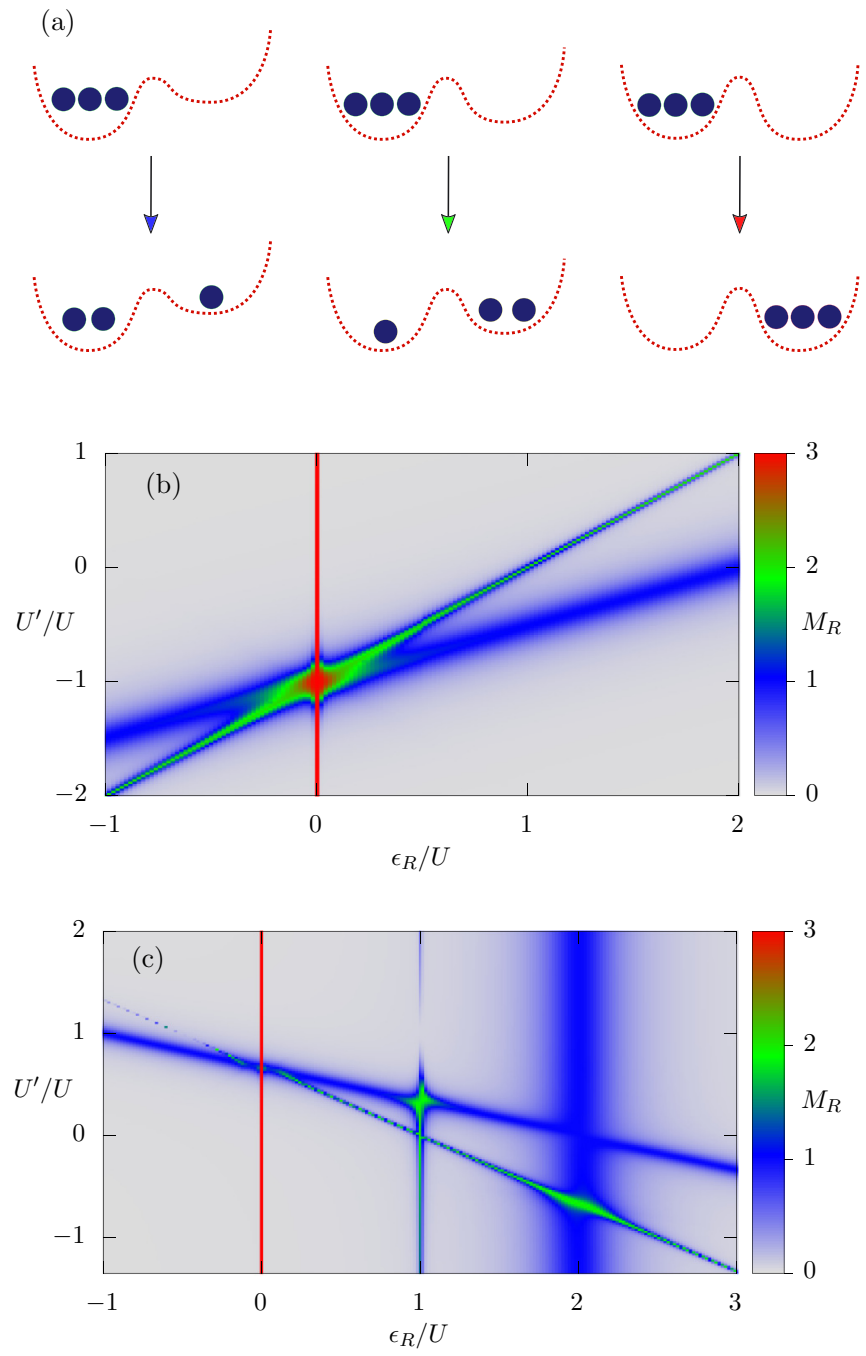

FIG. 4. Dynamics of three spin-1 bosons in a double well starting with all three on the left as schematically represented in (a). The diagrams in (b) and (c), respectively, for $S_{T}=3$ and $S_{T}=1$ are intensity maps of the maximum average occupation $M_{R}$ of the right-hand side after a long evolution time for $J / U=0.08$. Resonant single-particle (blue), pair (green), and triad tunnelings (red) occur in narrow regions of the $\left(\epsilon_{R}, U^{\prime}\right)$ parameter space.

first order in $J$, the only real dynamical process by which an atom goes to the other well is a single-particle conditional tunneling for $\epsilon_{R}=2\left(U+U^{\prime}\right)$. In addition, a second-order conditional tunneling occurs for $\epsilon_{R}=U+U^{\prime}$ in which a pair of atoms jumps to the empty well leaving a single atom on the left. With symmetric wells $\left(\epsilon_{R}=0\right)$, the only possible process is of third order in $J$ with the three atoms tunneling as a bound triad. In each of these cases, the initial and final states alternate periodically in time. This happens with very different time scales for the three processes, similar to what was discussed for two particles. A pictorial representation of these three processes is shown in Fig. 4(a). The resonance conditions appear as reasonably well-defined lines in the $U^{\prime}$ vs $\epsilon_{R}$ diagram of Fig. 4(b) where the colors represent the maximum average occupation $\left(M_{R}\right)$ of the right-hand side after a long evolution time. Here, as in the two-particle case, the line on which $M_{R} \simeq 1$ is wider due to off-resonance single 
atom visits to the other well. All processes are resonant at the crossing point $\left(\epsilon_{R}=0, U^{\prime}=-U\right)$.

Turning now to the low-spin case, we have a larger subspace because states with $S_{i}=0$ and 2 are allowed for $N_{i}=2$. Therefore, single-particle tunneling from the initialstate $|\{3,0\},\{1,0\}, 1\rangle$ generates the states $|\{2,1\},\{2,1\}, 1\rangle$ or $|\{2,1\},\{0,1\}, 1\rangle$ with resonance conditions $\epsilon_{R}=2 U-3 U^{\prime}$ and $\epsilon_{R}=2 U$, respectively. Similarly, second-order pair tunneling leads to the final state $|\{1,2\},\{1,2\}, 1\rangle$ if $\epsilon_{R}=\left(2 U-3 U^{\prime}\right) / 2$ or $|\{1,2\},\{1,0\}, 1\rangle$ if $\epsilon_{R}=U$. Triad tunneling remains the only real process for an unbiased double well. The $U^{\prime}$ vs $\epsilon_{R}$ diagram for $S_{T}=1$, shown in Fig. 4(c), is richer than the high-spin one due to the extra processes just described.

\section{CONCLUSIONS}

We have described various dynamical processes occurring for two or three spin-1 bosons in double wells that may present depth asymmetry. These bosons interact both through the usual repulsive Hubbard- $U$ term and a spin-dependent interaction that may be chosen to favor high or low spin. We focus on the strong-coupling limit, that is a relatively small single-particle tunneling amplitude so that dynamical processes are strongly suppressed except under very strict relationships between the system parameters. Since the target experimental realization consists of optically trapped cold atoms, the fundamental constraint is energy conservation. Then, resonant tunneling will only occur if lost or gained interaction energy is compensated by well-depth asymmetry affecting local single-particle levels.
Our initial state is chosen with all particles in one of the wells and in a defined total-spin quantum state. This is convenient since the time evolution after tunneling is "turned on" conserves the total spin. For symmetric wells, the particles must move together as a bound pair or triad, depending on the initial total number. The probability is low because it is a second- or third-order tunneling process, which means that these coupled motions between the two wells occur on a very large time scale. If the initially empty well is raised in energy, conditional single-particle or pair tunneling, respectively, become possible for a total number of two or three bosons in the double well.

The resonance diagrams of Figs. 3 and 4 show that the existence of a spin-dependent interaction (as well as its intensity and sign) can be experimentally determined from the tuned value of the depth difference between the two wells.

It is worth remarking that the dynamical processes analyzed here involve few-particle states that are highly entangled between left and right wells. The possibility of tuning the number of particles that move through the system and the time scale for this motion is potentially very important for applications in the promising field of atomtronics with possible impacts on the search for realizable quantum-computing systems.

\section{ACKNOWLEDGMENTS}

This work was supported in part by the Brazilian agencies Conselho Nacional de Desenvolvimento Científico e Tecnológico (CNPq) and Coordenação de Aperfeiçoamento de Pessoal de Nível Superior (CAPES).
[1] M. H. Anderson, J. R. Ensher, M. R. Matthews, C. E. Wieman, and E. A. Cornell, Observation of Bose-Einstein condensation in a dilute atomic vapor, Science 269, 198 (1995).

[2] K. B. Davis, M. O. Mewes, M. R. Andrews, N. J. van Druten, D. S. Durfee, D. M. Kurn, and W. Ketterle, Bose-Einstein Condensation in a Gas of Sodium Atoms, Phys. Rev. Lett. 75, 3969 (1995).

[3] D. C. McKay and B. DeMarco, Cooling in strongly correlated optical lattices: Prospects and challenges, Rep. Prog. Phys. 74, 054401 (2011).

[4] D. M. Stamper-Kurn and M. Ueda, Spinor Bose gases: Symmetries, magnetism, and quantum dynamics, Rev. Mod. Phys. 85, 1191 (2013).

[5] T. Ohmi and K. Machida, Bose-Einstein condensation with internal degrees of freedom in alkali atom gases, J. Phys. Soc. Jpn. 67, 1822 (1998).

[6] T.-L. Ho, Spinor Bose Condensates in Optical Traps, Phys. Rev. Lett. 81, 742 (1998).

[7] B. Juliá-Díaz, M. Melé-Messeguer, M. Guilleumas, and A. Polls, Spinor Bose-Einstein condensates in a double well: Population transfer and Josephson oscillations, Phys. Rev. A 80, 043622 (2009).

[8] M. Melé-Messeguer, S. Paganelli, B. Juliá-Díaz, A. Sanpera, and A. Polls, Spin-driven spatial symmetry breaking of spinor condensates in a double well, Phys. Rev. A 86, 053626 (2012).

[9] S. Trotzky, P. Cheinet, S. Fölling, M. Feld, U. Schnorrberger, A. M. Rey, A. Polkovnikov, E. A. Demler, M. D. Lukin, and
I. Bloch, Time-resolved observation and control of superexchange interactions with ultracold atoms in optical lattices, Science 319, 295 (2008).

[10] Ö. E. Müstecaplığlu, W.Zhang, and L. You, Quantum dynamics of a spin-1 condensate in a double-well potential, Phys. Rev. A 75, 023605 (2007).

[11] I. Bloch, Ultracold quantum gases in optical lattices, Nat. Phys. 1, 23 (2005).

[12] J. Sebby-Strabley, B. L. Brown, M. Anderlini, P. J. Lee, W. D. Phillips, J. V. Porto, and P. R. Johnson, Preparing and Probing Atomic Number States with an Atom Interferometer, Phys. Rev. Lett. 98, 200405 (2007).

[13] C. Weitenberg, M. Endres, J. F. Sherson, M. Cheneau, P. Schau, T. Fukuhara, I. Bloch, and S. Kuhr, Single-spin addressing in an atomic Mott insulator, Nature (London) 471, 319 (2011).

[14] I. Bloch, J. Dalibard, and S. Nascimbène, Quantum simulations with ultracold quantum gases, Nat. Phys. 8, 267 (2012).

[15] M. Endres, M. Cheneau, T. Fukuhara, C. Weitenberg, P. Schauß, C. Gross, L. Mazza, M. C. Bañuls, L. Pollet, I. Bloch, and S. Kuhr, Single-site- and single-atom-resolved measurement of correlation functions, Appl. Phys. B 113, 27 (2013).

[16] A. Albert, C. Robens, W. Alt, S. Brakhane, M Karski, R. Reimann, A. Widera, and D. Meschede, Super-resolution microscopy of single atoms in optical lattices, New J. Phys. 18, 053010 (2016).

[17] M. Boll, T. A. Hilker, G. Salomon, A. Omran, J. Nespolo, L. Pollet, I. Bloch, and C. Gross, Spin- and density-resolved 
microscopy of antiferromagnetic correlations in Fermi-Hubbard chains, Science 353, 1257 (2016).

[18] H. N. Dai, B. Yang, A. Reingruber, X. F. Xu, X. Jiang, Y. A. Chen, Z. S. Yuan, and J. W. Pan, Generation and detection of atomic spin entanglement in optical lattices, Nat. Phys. 12, 783 (2016).

[19] C. Gross and I. Bloch, Quantum simulations with ultracold atoms in optical lattices, Science 357, 995 (2017).

[20] E. Braaten and H.-W. Hammer, Universality in few-body systems with large scattering length, Phys. Rep. 428, 259 (2006).

[21] D. Blume, Few-body physics with ultracold atomic and molecular systems in traps, Rep. Prog. Phys. 75, 046401 (2012).

[22] A. Wagner, C. Bruder, and E. Demler, Spin-1 atoms in optical superlattices: Single-atom tunneling and entanglement, Phys. Rev. A 84, 063636 (2011).

[23] D. W. S. Carvalho, A. Foerster, and M. A. Gusmão, Ground states of spin-1 bosons in asymmetric double wells, Phys. Rev. A 91, 033608 (2015).

[24] B. T. Seaman, M. Krämer, D. Z. Anderson, and M. J. Holland, Atomtronics: Ultracold-atom analogs of electronic devices, Phys. Rev. A 75, 023615 (2007).

[25] R. A. Pepino, J. Cooper, D. Z. Anderson, and M. J. Holland, Atomtronic Circuits of Diodes and Transistors, Phys. Rev. Lett. 103, 140405 (2009).

[26] R. A. Pepino, J. Cooper, D. Meiser, D. Z. Anderson, and M. J. Holland, Open quantum systems approach to atomtronics, Phys. Rev. A 82, 013640 (2010).

[27] F. Serwane, G. Zurn, T. Lompe, T. B. Ottenstein, A. N. Wenz, and S. Jochim, Deterministic preparation of a tunable few-fermion system, Science 332, 336 (2011).

[28] S. Murmann, A. Bergschneider, V. M. Klinkhamer, G. Zürn, T. Lompe, and S. Jochim, Two Fermions in a Double Well:
Exploring a Fundamental Building Block of the Hubbard Model, Phys. Rev. Lett. 114, 080402 (2015).

[29] K. Winkler, G. Thalhammer, F. Lang, R. Grimm, J. H. Denschlag, A. J. Daley, A. Kantian, H. P. Buchler, and P. Zoller, Repulsively bound atom pairs in an optical lattice, Nature (London) 441, 853 (2006).

[30] S. Fölling, S. Trotzky, P. Cheinet, M. Feld, R. Saers, A. Widera, T. Müller, and I. Bloch, Direct observation of second-order atom tunnelling, Nature (London) 448, 1029 (2007).

[31] S. Zöllner, H.-D. Meyer, and P. Schmelcher, Tunneling dynamics of a few bosons in a double well, Phys. Rev. A 78, 013621 (2008).

[32] P. Cheinet, S. Trotzky, M. Feld, U. Schnorrberger, M. MorenoCardoner, S. Fölling, and I. Bloch, Counting Atoms Using Interaction Blockade in an Optical Superlattice, Phys. Rev. Lett. 101, 090404 (2008).

[33] D. Jaksch, C. Bruder, J. I. Cirac, C. W. Gardiner, and P. Zoller, Cold Bosonic Atoms in Optical Lattices, Phys. Rev. Lett. 81, 3108 (1998).

[34] A. Imambekov, M. Lukin, and E. Demler, Spin-exchange interactions of spin-one bosons in optical lattices: Singlet, nematic, and dimerized phases, Phys. Rev. A 68, 063602 (2003).

[35] M. Greiner, O. Mandel, T. Esslinger, T. W. Hänsch, and I. Bloch, Quantum phase transition from a superfluid to a Mott insulator in a gas of ultracold atoms, Nature (London) 415, 39 (2002).

[36] S. Zöllner, H.-D. Meyer, and P. Schmelcher, Ultracold fewboson systems in a double-well trap, Phys. Rev. A 74, 053612 (2006).

[37] S. Zöllner, H.-D. Meyer, and P. Schmelcher, Few-Boson Dynamics in Double Wells: From Single-Atom to Correlated Pair Tunneling, Phys. Rev. Lett. 100, 040401 (2008). 